\title{
Diabetes Mellitus Is an Important Predictor for Hospitalization and Mortality From the COVID-19 Infection: A Substantial Interface Between Two Outbreaks
}

\author{
Khaled K. Aldossari
}

\begin{abstract}
Individuals with comorbidities such as diabetes mellitus (DM) are more likely to develop the complications and fatalities from COVID-19. Although researchers have performed studies across the world to evaluate the interaction between DM and COVID-19 with a specific focus on DM as a predictor or determinant for COVID-19, these studies are not synthesized collectively to provide stronger evidence. Hence we aimed to review the studies that have assessed whether diabetes is a predictor or determinants for morbidity and death among patients with COVID-19. Different articles were searched from databases such as Google Scholar and PubMed. We undertook a narrative review of full-text research articles that were published in the English language both in developed and developing countries, with a specific focus on diabetes and COVID-19. All primary research articles were examined for information pertinent to the objective. We also reviewed all references of the eligible article to avoid missing any article relevant to the topic of interest. The existing evidence suggests that diabetic patients are at increased risk for adverse outcomes including death from COVID-19. The synthesis of the review revealed that evidence regarding diabetes as a predictor or determinants for morbidity and death among patients with COVID-19 is mixed. Individuals with diabetes are at risk of infections such as COVID-19. Thus, most of the studies have identified DM as a predictor for mortality in patients with COVID-19. In contrast, there are very few studies that reported contrary findings. Individuals with DM are at higher risk of catching infections such as COVID-19. This risk can be decreased, though not eradicated, by maintaining optimum levels of glucose in the blood. Indeed, diabetes was an important predictor for morbidity and death among patients with COVID-19. Therefore, these patients should take extra precautionary measures with good glycemic control and frequent monitoring of blood glucose.
\end{abstract}

Manuscript submitted July 13, 2020, accepted July 20, 2020

Published online August 26, 2020

Family and Community Medicine Department, College of Medicine, Prince Sattam Bin Abdulaziz University, Al-Kharj, Saudi Arabia.

Email: Khalid317@gmail.com

doi: https://doi.org/10.14740/jem665
Keywords: Diabetes mellitus; Risk factor; Mortality; Morbidity; COVID-19

\section{Introduction}

Coronavirus disease 2019 (COVID-19) has spread quickly across the globe $[1,2]$. COVID-19 can lead to severe acute respiratory tract infection among infected individuals, and it is human to human transmission through saliva, nasal droplets, hands, and surface contacts [3, 4]. Since the end of 2019, this newly identified deadly illness has spread swiftly through China and the remaining world. The rapidly spreading pandemic of COVID-19 has caused substantial morbidity and mortality $[5,6]$.

By March 16, 2020, the proportion of cases of COVID-19 had increased drastically outside China, and the number of affected countries reporting confirmed cases to the World Health Organization (WHO) increased drastically within weeks and then within a few days. Ever since the first case of COVID-19 was detected in Wuhan in the Hubei district of China in late November 2019, the virus has spread rapidly [7], owing to its highly contagious nature, across countries and continents [5, 6]. With China reporting a significant decline in the incidence of COVID-19 following these measures, countries around the world were quick to follow suit [8]. On March 16, 2020, the WHO declared the COVID-19 to be a pandemic which had then affected 168,000 individuals and claimed 6,500 lives across the globe [9].

An interactive web-based dashboard managed by the Center for Systems Science and Engineering (CSSE) at Johns Hopkins University, MD, USA estimates the total number of cases to be $5,270,244$, which includes $2,087,326$ recoveries and 340,116 deaths as of May 23, 2020 [10]. The COVID-19 global health pandemic has spoiled the health status, as well as the quality of life of many individuals across the world. The highly contagious nature of the virus and its tendency to spread from person to person via aerosol droplets meant that the only way to curb its swift spread was to impose containment measures by enforcing nationwide lockdowns and travel restrictions 
and by encouraging social distancing [11]. These measures taken by different governments have affected the economies and health system to a greater extent.

Individuals with health conditions such as diabetes mellitus (DM), hypertension (HTN), and severe obesity are more likely to develop complications and fatalities from COVID-19. Although a significant number of patients suffering from these comorbidities might have remained asymptomatic, but they have a higher potential to transmit the virus, which in turn could increase the number of symptomatic individuals that need to be hospitalized. Considering the burden of non-communicable and degenerative diseases such as DM, HTN, and severe obesity both in developed and developing countries and the substantially higher risk for COVID-19 and its adverse outcomes in patients with these comorbidities, it is possible the outbreak might have the potential to cause a higher rate of disease and death among these patients. Moreover, DM is one of the primary causes of sickness globally and is expected to upsurge significantly in the coming future [12]. Numerous studies have demonstrated a higher predisposition of diabetic patients to some infectious diseases such as Staphylococcus aureus and Mycobacterium tuberculosis [13, 14]. This might be because of the weak and dysregulated immune system of the patients suffering from DM [15]. However, it is proven that DM predisposes to some infections and mortality from those infections, but it is still not clear whether DM is a predictor or determinant for the prognosis of COVID-19 [13, 14]. Furthermore, it has been specified in the literature that higher levels of blood glucose and DM are independent risk factors for death and morbidity in the diagnosed patients of COVID-19 [16].

Although researchers have performed studies across the world to evaluate the interaction between DM and COVID-19 with a specific focus on DM as a risk factor for COVID-19, these studies are not synthesized collectively to provide stronger evidence. Therefore, there is a need to undertake a review to understand the interface between DM and COVID-19. Hence the overarching objective is to review, appraise, and synthesize findings from different studies conducted on assessing the relationship between DM and COVID-19. More specifically, we aimed to review the studies that have assessed whether diabetes is a predictor or determinant to affect the prognosis of COVID-19. This will help to guide clinicians and endocrinologists to provide appropriate treatment to the patients suffering from DM, and also to guide these patients to take extra precautionary measures to prevent themselves from this deadly disease.

\section{Literature Retrieval}

We searched articles from databases such as Google Scholar and PubMed. For this narrative review, the authors undertook controlled vocabulary and text-word searches in these two databases. All articles published on the topic of DM and COVID-19 were included in this review. We searched all the articles by using search terms such as "diabetes mellitus", "DM" "DM and COVID-19", "diabetes mellitus and COVID-19" "interaction between DM and COVID-19", "diabetes mellitus as a risk factor for COVID-19" and "diabetes mellitus as a predictor of COVID-19". Studies were limited to human studies reported in the English language, and studies conducted both in developing and developed countries were included. We examined all primary and original articles pertinent to our objective while conducting this review. Given the timing of pandemic from COVID-19, we limited our search between 2019 and 2020 and applied filter on the time period while searching the articles in the databases. We undertook a search of electronic databases by using a snowball sampling technique consisting of backward and forward reference searching of articles to identify the eligible articles. Besides, we also reviewed all references of the eligible article to avoid missing any article germane to the topic of interest. After searching for different databases and following the above criteria, we reviewed full-text articles of the studies that highlighted the interface or interaction between DM and COVID-19.

\section{Findings of the Narrative Review}

The findings of the narrative review are divided into two main sections. The first section gives a brief review of all the studies included in the review and whose objective was to assess DM as a predictor or determinant for COVID-19 disease and death. The second section gives a series of potential recommendations and specific measures to be taken by the patients suffering from DM but with and without COVID-19 infection.

\section{Section 1: DM as a predictor or risk factor for COVID-19 morbidity and mortality}

Generally, there is a scarcity of data about COVID-19 in patients with DM at the moment. However, the evidence from available studies is summarized below in detail. A retrospective hospital-based study was conducted on 174 patients with COVID-19 infection who were admitted to Wuhan Union Hospital in China in February. The authors in their study analyzed data on demographic characteristics, history of exposure, signs and symptoms, medical history, laboratory reports, computed tomography (CT) scans of the chest, and the treatment modalities from medical records of the patients. On average the patients with COVID-19 in this study were 59 years old and around half of the patients were males [17]. The authors found that the most common underlying comorbidities were HTN and DM. Also, patients with DM had more cardiovascular disease and less fever when compared with patients without diabetes. However, there were no significant differences in gender and age and death rates between the two groups [17]. Furthermore, the authors also reported that as opposed to the patients without diabetes, patients with diabetes were elderly, had more symptoms such as nausea and vomiting and a higher rate of death. However, there were no significant differences in sex and other symptoms, time for follow-up, and the time from the beginning of symptoms to admission in the hospital between patients with and without DM [17]. Overall, whether interference from other chronic diseases such as HTN or cardiovascu- 
lar diseases is existed or nor, authors found that severe acute respiratory syndrome coronavirus 2 (SARS-CoV-2) pneumonia patients with DM are more severe than those without DM. This was assessed from markers such as inflammatory factors or hypercoagulability, organ damage, and they are at higher risk of progressing into a worse prognosis [17].

Another study was conducted in Wuhan, China on 26 cases with fatalities to reveal the serious comorbidities [18]. This study demonstrated that the common comorbidities were DM $(42.3 \%)$, chronic bronchitis $(19.2 \%)$, cerebral infarction $(15.4 \%)$, HTN (53.8\%), coronary heart disease (CHD) $(19.2 \%)$, and Parkinson's disease $(7.7 \%)$. This study also revealed that DM was found in $42.3 \%$ of the total fatalities due to COVID-19, which was the second most common disease found in the patients of COVID-19, thus indicating the higher burden of DM in these patients after HTN [18]. The authors from this analysis concluded that the comorbidities such as DM, HTN, CHD, cerebral infarction, and chronic bronchitis were found to be severe factors that led to the mortality in the patients infected with COVID-19. This further supports the evidence that diabetes could be one of the predictors for poor prognosis in patients with COVID-19 [18].

Likewise, one more study was conducted in 150 patients in Wuhan, China on confirmed patients of COVID-19. The authors used the database of Jin Yin-tan Hospital and Tongji Hospital and undertook a multicenter study of 68 death cases and 82 discharged cases who were confirmed cased of SARSCoV-2 on the laboratory testing [19]. Overall, the authors found a positive correlation between the number of comorbidities and mortality from COVID-19 thus indicating that underlying comorbid could be a potential determinant for death in the patients of COVID-19 [19].

In addition, the Chinese Centre for Disease Control and Prevention published a report of 72,314 cases of COVID-19 that revealed an increased rate of fatality in diabetic patients $(7.3 \%)$ when compared to the overall mortality of $2.3 \%$ [20]. More specifically, this report showed that there was no mortality in patients aged 9 years and younger [20]. However, the case fatality rate was $8.0 \%$ in older patients $(<80$ years old) and $14.8 \%$ in those aged 80 years and older. Moreover, the study also revealed that the case fatality rate was $49.0 \%$ among severely ill cases, and it was higher among those with prior comorbidities. For example, case fatality rate was $5.6 \%$ for cancer, $6.0 \%$ and $6.3 \%$ for chronic respiratory disease and HTN, $7.3 \%$ for diabetes, and $10.5 \%$ for cardiovascular disease [20]. Among the 44,672 cases, 3.8\% were health workers, and $63 \%$ in Wuhan only. This indicates an increased ratio of fatalities among patients suffering from DM when compared to their counterparts [20].

Additionally, an observational report that included 1,099 confirmed COVID-19 patients specified that in 173 severe cases, there were comorbidities such as high blood pressure (23.7\%), DM (16.2\%), and cardiovascular diseases (9\%) [21]. The emerging data from a large observational study specifies that diabetic patients indeed are at higher risk of getting COVID-19 infection [21]. Similarly, authors in another study analyzed electronic medical records such as demographics, clinical manifestation, comorbidities, laboratory data, and radiological materials of 140 hospitalized and confirmed COV-
ID-19 patients [22]. In this particular study of 140 patients, authors found that 30\% had HTN and $12 \%$ had diabetes thus further supporting the evidence of DM as a risk factor for COVID-19 [22].

In contrast to the above studies, one more study conducted in China found contrary results to the above-mentioned study, and this study was conducted on 140 diagnosed cases of COVID-19, where around 82 patients were classified into nonsevere patients and 58 were classified as severe cases at the time of admission in the hospital. On average, the participants were 57 years old, and most of them were older patients [18]. The study found that DM (12.1\%) and HTN (30.0\%) were the highly prevalent comorbidities among these patients suffering from COVID-19. Overall, authors in this study did not find diabetes as a predictor for severe disease course in patients suffering from COVID-19, thus providing controversial results when compared to the other studies [18]. This is consistent with the analysis of 11 studies, which reviewed the laboratory abnormalities among patients suffering from COVID-19. This analysis concludes that elevated blood glucose or diabetes cannot be considered as a risk factor of severe disease [23].

\section{Section 2: precautionary measures need to be taken by the patients with DM}

Patients suffering from DM need to take some precautionary measures either to prevent themselves from getting infected by COVID-19, or to avert the spread of the infection of COVID-19 if they have already caught the disease. These measures are divided into two broader categories as discussed below.

\section{Measures for prevention of COVID-19 in patients with DM}

Given the nature of the disease and the weak immune system of patients with DM, these patients must take some specific measures to prevent themselves from this deadly disease in the future. Firstly, patients suffering from DM must maintain their blood glucose at the optimal levels, as it will help them in plummeting the danger of infection. Besides, it is also important for these patients to frequently monitor their blood glucose levels and self-monitor their glucose levels in the blood. This, in turn, will help them to lessen the risk of secondary bacterial pneumonia. Secondly, people with DM and existing comorbid such as cardiovascular and renal disease need exceptional care, and patients should make efforts to stabilize their cardiac and renal status to avoid getting an infection. Thirdly, these patients with DM should take care of their nutrition and protein intake to maintain the required amount of minerals and vitamins in the body, otherwise, they can catch infection easily. Fourthly, these patients need to exercise regularly to augment immunity; however, it might be judicious to be extra careful and patients should avoid places such as swimming pools or gymnasia with large crowds. Lastly, patients suffering from DM should get vaccinations against influenza and pneumonia as it will reduce the likelihood of getting bacterial pneumonia after getting a respiratory viral infection. 
Precautionary measures by the patients suffering from DM and COVID-19

If a diabetic patient develops COVID-19, he or she can take some of the control measures to avert the disease spread and also to decrease the damage to their bodies. First of all, if a person with diabetes develops symptoms of COVID-19, the right health specialist in a particular country needs to be cognizant as testing for COVID-19 is not available at all settings and places. Secondly, it is very important to isolate the affected person for 2 weeks or till the symptoms disappear, and patients suffering from DM must follow the guidelines endorsed by their respective countries. In addition, most of the individuals can be treated at home due to mild symptoms; therefore, patients need to hydrate themselves with proper treatment such as using acetaminophen for fever and symptomatic treatment such as steam inhalation to get relief from running nose. Moreover, patients with DM should measure their blood glucose frequently if fever with hyperglycemia occurs because there is a possibility to make frequent changes in dosage and correctional bolus to maintain normoglycemia. It is imperative for the patients suffering from DM to stop taking antidiabetic medication that can reduce the volume of blood and cause hypoglycemia. Besides, the physicians might need to reduce the dosage of oral antidiabetic medication if necessary. Patients may need more frequent monitoring of blood glucose and drug adjustment as per the guidelines and advice by their physicians. Lastly, blood glucose should be frequently monitored in hospitalized patients suffering from severe COVID-19 infection. Oral antidiabetic medication such as metformin and sodium-glucose cotransporter-2 inhibitors should not be given to the patients as insulin is considered as the ideal mode of therapy to control hyperglycemia in hospitalized and ill individuals suffering from DM.

\section{Conclusions}

Emerging evidence alludes that diabetic patient are at higher risk for getting adverse outcomes from COVID-19 including a higher rate of mortality. Analogous confirmation of risk among diabetic individuals has been reported for the two former COVID infections, severe acute respiratory syndrome that affected numerous individuals in 2002 and disturbing thousands of persons, mostly in Asia and the Middle East [24], and the Middle East respiratory syndrome (MERS) in 2012 that affected more than 2,000 persons, largely in the cities of Saudi Arabia [25]. Overall, the summary of the review revealed that evidence regarding diabetes as a predictor or determinant for morbidity and fatality among patients with COVID-19 is mixed.

The majority of the evidence comes from studies conducted in China, and there is a need for more evidence before making any conclusion. However, most of the studies have identified DM as a risk factor for mortality in patients with COVID-19. Thus, diabetes could be regarded as a potential predictor or determinant for the consequences of COVID-19; therefore, additional attention should be provided to individuals suffering from DM. Since individuals with diabetes have a higher probability of getting infections such as COVID-19, therefore, this probability of getting an infection can be decreased, however, completely not removed, by maintaining glycemic levels in the body. All individuals with diabetes are advised to get vaccinations against pneumonia and influenza. Besides, these patients should take extra precautionary measures with good glycemic control and frequent monitoring of blood glucose.

\section{Acknowledgments}

This publication was supported by the Deanship of Scientific Research, Prince Sattam Bin Abdulaziz University, Al-kharj, Saudi Arabia.

\section{Financial Disclosure}

This research did not receive any specific grant from funding agencies in the public, commercial, or not-for-profit sectors.

\section{Conflict of Interest}

None to declare.

\section{Author Contributions}

The author wrote and read the manuscript.

\section{Data Availability}

The authors declare that data supporting the findings of this study are available within the article.

\section{References}

1. Chen Y, Liu Q, Guo D. Emerging coronaviruses: Genome structure, replication, and pathogenesis. J Med Virol. 2020;92(4):418-423.

2. Tu H, Tu S, Gao S, Shao A, Sheng J. Current epidemiological and clinical features of COVID-19; a global perspective from China. J Infect. 2020;81(1):1-9.

3. Khurshid Z, Asiri FYI, Al Wadaani H. Human saliva: non-invasive fluid for detecting novel coronavirus (2019nCoV). Int J Environ Res Public Health. 2020;17(7).

4. Peng X, Xu X, Li Y, Cheng L, Zhou X, Ren B. Transmission routes of 2019-nCoV and controls in dental practice. Int J Oral Sci. 2020;12(1):9.

5. Nishiura H, Jung SM, Linton NM, Kinoshita R, Yang Y, Hayashi K, Kobayashi T, et al. The extent of transmission of novel coronavirus in Wuhan, China, 2020. J Clin Med. 2020;9(2).

6. Khachfe HH, Chahrour M, Sammouri J, Salhab H, Makki 
BE, Fares M. An Epidemiological Study on COVID-19: A Rapidly Spreading Disease. Cureus. 2020;12(3):e7313.

7. Liu SL, Saif L. Emerging viruses without borders: the Wuhan coronavirus. Viruses. 2020;12(2).

8. Sjodin H, Wilder-Smith A, Osman S, Farooq Z, Rocklov J. Only strict quarantine measures can curb the coronavirus disease (COVID-19) outbreak in Italy, 2020. Euro Surveill. 2020;25(13).

9. Mahase E. Covid-19: WHO declares pandemic because of "alarming levels" of spread, severity, and inaction. BMJ. 2020;368:m1036.

10. Dong E, Du H, Gardner L. An interactive web-based dashboard to track COVID-19 in real time. Lancet Infect Dis. 2020;20(5):533-534.

11. Peng F, Tu L, Yang Y, Hu P, Wang R, Hu Q, Cao F, et al. Management and treatment of COVID-19: the Chinese experience. Can J Cardiol. 2020;36(6):915-930.

12. Knapp S. Diabetes and infection: is there a link? A minireview. Gerontology. 2013;59(2):99-104.

13. Shah BR, Hux JE. Quantifying the risk of infectious diseases for people with diabetes. Diabetes Care. 2003;26(2):510-513.

14. Muller LM, Gorter KJ, Hak E, Goudzwaard WL, Schellevis FG, Hoepelman AI, Rutten GE. Increased risk of common infections in patients with type 1 and type 2 diabetes mellitus. Clin Infect Dis. 2005;41(3):281-288.

15. Joshi N, Caputo GM, Weitekamp MR, Karchmer AW. Infections in patients with diabetes mellitus. N Engl J Med. 1999;341(25):1906-1912.

16. Yang JK, Feng Y, Yuan MY, Yuan SY, Fu HJ, Wu BY, Sun $\mathrm{GZ}$, et al. Plasma glucose levels and diabetes are independent predictors for mortality and morbidity in patients with SARS. Diabet Med. 2006;23(6):623-628.

17. Guo W, Li M, Dong Y, Zhou H, Zhang Z, Tian C, Qin
$\mathrm{R}$, et al. Diabetes is a risk factor for the progression and prognosis of COVID-19. Diabetes Metab Res Rev. 2020:e3319.

18. Deng SQ, Peng HJ. Characteristics of and Public Health Responses to the Coronavirus Disease 2019 Outbreak in China. J Clin Med. 2020;9(2).

19. Ruan Q, Yang K, Wang W, Jiang L, Song J. Clinical predictors of mortality due to COVID-19 based on an analysis of data of 150 patients from Wuhan, China. Intensive Care Med. 2020;46(5):846-848.

20. Wu Z, McGoogan JM. Characteristics of and important lessons from the coronavirus disease 2019 (COVID-19) outbreak in China: summary of a report of 72314 cases from the Chinese Center for Disease Control and Prevention. JAMA. 2020.

21. Guan WJ, Ni ZY, Hu Y, Liang WH, Ou CQ, He JX, Liu L, et al. Clinical characteristics of coronavirus disease 2019 in China. N Engl J Med. 2020;382(18):1708-1720.

22. Zhang JJ, Dong X, Cao YY, Yuan YD, Yang YB, Yan YQ, Akdis CA, et al. Clinical characteristics of 140 patients infected with SARS-CoV-2 in Wuhan, China. Allergy. 2020;75(7):1730-1741.

23. Lippi G, Plebani M. Laboratory abnormalities in patients with COVID-2019 infection. Clin Chem Lab Med. 2020;58(7):1131-1134.

24. Huang YT, Lee YC, Hsiao CJ. Hospitalization for ambulatory-care-sensitive conditions in Taiwan following the SARS outbreak: a population-based interrupted time series study. J Formos Med Assoc. 2009;108(5):386-394.

25. Morra ME, Van Thanh L, Kamel MG, Ghazy AA, Altibi AMA, Dat LM, Thy TNX, et al. Clinical outcomes of current medical approaches for Middle East respiratory syndrome: A systematic review and meta-analysis. Rev Med Virol. 2018;28(3):e1977. 